\title{
Atrial fibrillation in healthy adolescents after highly caffeinated beverage consumption: two case reports
}

\author{
Jennifer R Di Rocco ${ }^{1}$, Adelaide During ${ }^{2}$, Peter J Morelli ${ }^{3 *}$, Marybeth Heyden ${ }^{3}$, Thomas A Biancaniello ${ }^{3}$
}

\begin{abstract}
Introduction: Energy drinks and highly caffeinated drinks comprise some of the fastest growing products of the beverage industry, often targeting teenagers and young adults. Cardiac arrhythmias in children related to high caffeine consumption have not been well described in the literature. This case series describes the possible association between the consumption of highly caffeinated drinks and the subsequent development of atrial fibrillation in the adolescent population.
\end{abstract}

Case presentations: We report the cases of two Caucasian adolescent boys of 14 and 16 years of age at the time of presentation, each without a significant cardiac history, who presented with palpitations or vague chest discomfort or both after a recent history of excessive caffeine consumption. Both were found to have atrial fibrillation on electrocardiogram; one patient required digoxin to restore a normal sinus rhythm, and the other self-converted after intravenous fluid administration.

Conclusion: With the increasing popularity of energy drinks in the pediatric and adolescent population, physicians should be aware of the arrhythmogenic potential associated with highly caffeinated beverage consumption. It is important for pediatricians to understand the lack of regulation in the caffeine content and other ingredients of these high-energy beverages and their complications so that parents and children can be educated about the risk of cardiac arrhythmias with excessive energy drink consumption.

\section{Introduction}

Atrial fibrillation is extremely rare in the pediatric population, almost always occurring in association with structural heart disease, such as rheumatic mitral valve disease, congenital heart disease with dilated atria, and rarely, as a complication of intra-atrial surgery [1]. Patients may present with palpitations, dyspnea, fatigue, light-headedness, or syncope. The electrocardiogram is characterized by disorganized atrial activity without discrete $\mathrm{P}$ waves. The ventricular response is often irregularly irregular. Without a prior cardiac or family history, other inciting causes such as thyrotoxicosis, infectious pericarditis, and pulmonary emboli should be considered in the previously healthy child presenting with newonset atrial fibrillation [2].

\footnotetext{
* Correspondence: peter.morelli@stonybrook.edu

${ }^{3}$ Stony Brook University Department of Pediatric Cardiology, HSC T-11, 040, Stony Brook, NY, 11794-8111, USA

Full list of author information is available at the end of the article
}

Exogenous causes of atrial fibrillation through a substrate such as caffeine have not been widely reported in the literature, especially in the pediatric population. A large-scale Danish study evaluating adult human caffeine consumption and arrhythmias did not find a higher risk of atrial fibrillation or flutter with variable oral consumption of caffeine from everyday sources [3]. A controlled trial of escalating doses of caffeine in dogs surprisingly found that serum caffeine actually decreased the propensity for atrial fibrillation [4]; another canine trial demonstrated an increase in cardiac arrhythmias with high doses of caffeine administered [5]. A recent case report outlined a correlation between prolonged inhaled salbutamol and concurrent chocolate abuse, leading to an atrial arrhythmia in an adult, postulating that the caffeine in the chocolate coupled with the short-acting beta agonist triggered the arrhythmia [6]. Another case report described a 58-year-old man with atrial fibrillation and a dilated cardiomyopathy, which

\section{Ciomed Central}


resolved when he discontinued his excessive caffeine consumption [7].

Caffeine is a natural stimulant found in tea leaves, coffee beans, and cacao, and is one of the most popular psychoactive substances used today. Caffeine causes central and peripheral nervous system stimulation through antagonism of adenosine receptors and also has dopaminergic properties, which lend to its addictive potential [8]. The half-life of caffeine in a normal healthy adult is estimated to be from 2.5 to 10 hours, depending on the individual. Long-term consumption of caffeine or consumption of large amounts of caffeine will prolong its half-life $[9,10]$. The US Food and Drug Administration deems, "caffeine is generally recognized as safe when used in cola-type beverages up to a level of 0.02 percent" [11]. The population as a whole has variable sensitivity to the stimulant effects of caffeine; one's tolerance and dependence on caffeine seem to be somewhat heritable and may be linked to genetic polymorphisms [8]. The physiologic and psychological effects of caffeine have been studied in adults but have not been systematically analyzed in children [8].

Energy drinks and highly caffeinated drinks comprise some of the fastest-growing products of the beverage industry, often targeting teenagers and young adults $[8,12]$. This case series describes the possible association between the consumption of highly caffeinated drinks and the development of cardiac arrhythmias, specifically atrial fibrillation, in the adolescent population. We report two cases of atrial fibrillation in healthy adolescent boys after the consumption of energy drinks.

\section{Case presentation}

\section{Case 1}

A 14-year-old Caucasian boy with no significant past medical history presented with persistent "heart fluttering" two hours after a running race. He denied recent illness and denied drug ingestion, but reported drinking an unknown quantity of a highly caffeinated drink the day before. He also reported drinking a Red Bull ${ }^{\mathrm{TM}}$ energy drink five days before admission and feeling the same fluttering sensation. His physical examination revealed an irregularly irregular heart rate at approximately 130 beats per minute with a $1 / 6$ vibratory systolic ejection murmur at the left lower sternal border. Thyroid-function tests and serum calcium were normal. His electrocardiogram (ECG) showed narrow-complex tachycardia with atrial fibrillation and occasional atrial flutter (Figure 1). Cardiac ECG revealed a structurally normal heart without thrombus. He was treated with one dose of digoxin as a partial load at $7.5 \mu \mathrm{g} / \mathrm{kg}$ and quickly converted to normal sinus rhythm with a heart rate of 70 to 80 beats per minute (Figure 2). On followup examination in cardiology clinic one month later, the patient had a normal cardiac examination, a normal ECG, and no further symptoms of arrhythmia.

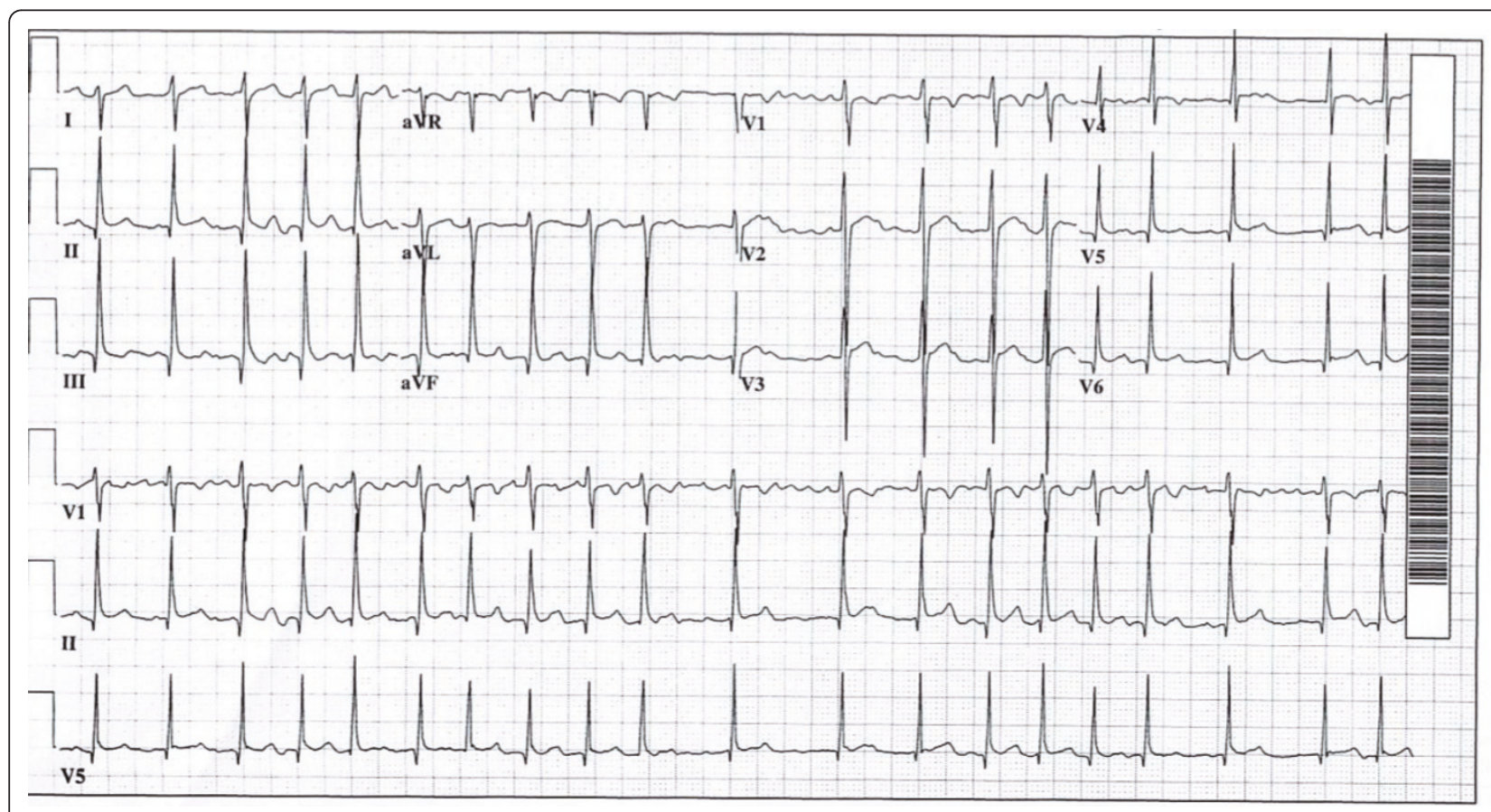

Figure 1 Electrocardiogram showing narrow-complex tachycardia with atrial fibrillation and occasional atrial flutter with irregularly irregular ventricular response. Heart rate, 122 beats per minute; QRS, 88 ms; QT, 433 ms. 


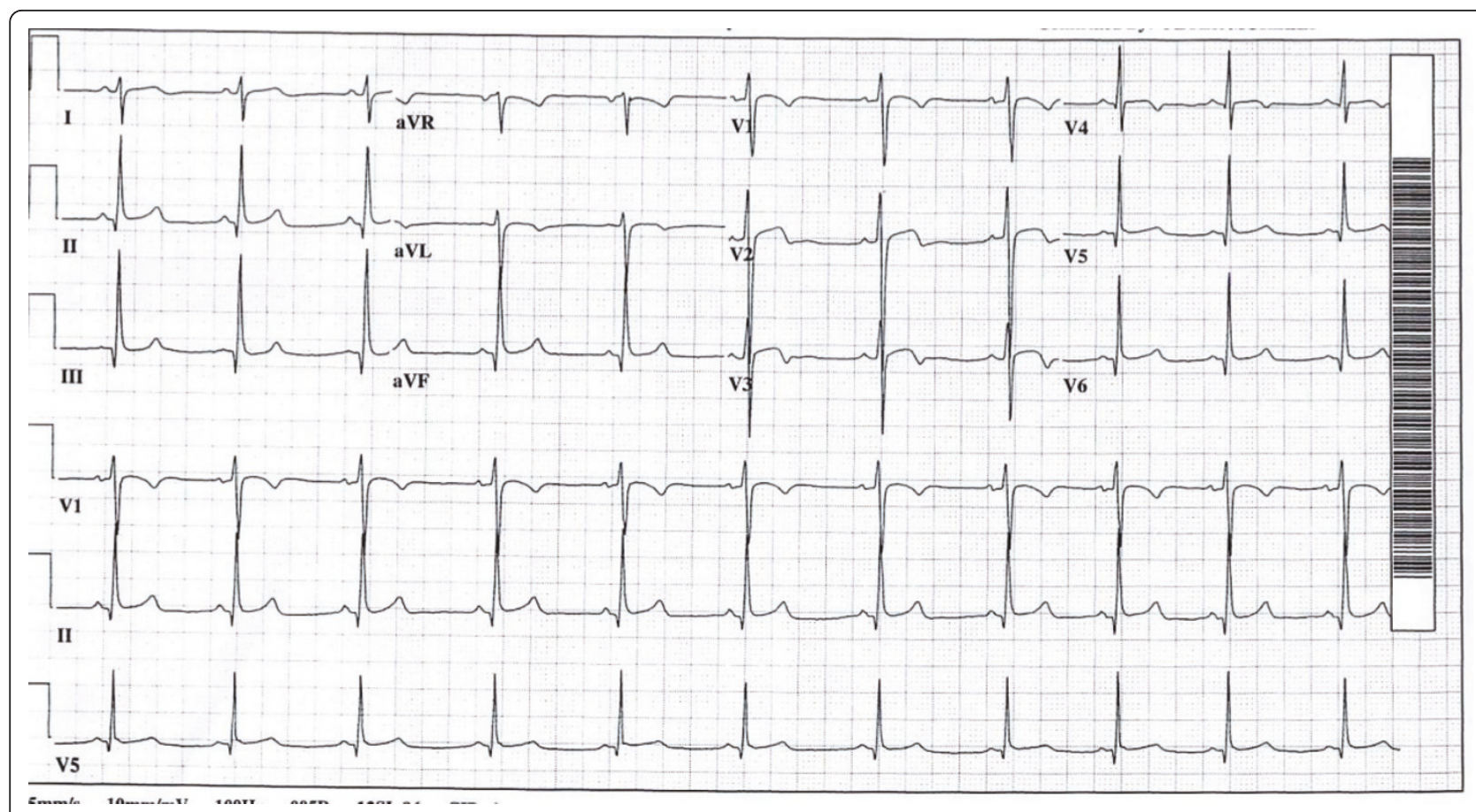

Figure 2 ECG showing normal sinus rhythm restored to 65 beats per minute after a dose of digoxin. PR interval, 129 ms; QRS, 100 ms; $\mathrm{QT}_{\mathrm{C}}, 398 \mathrm{~ms}$.

\section{Case 2}

A 16-year-old Caucasian boy with a history of attention-deficit hyperactivity disorder, asthma, and allergies presented to the emergency department with intoxication and vomiting after falling and sustaining minor head trauma. He had ingested an unknown quantity of Red BullTM mixed with vodka at a party. He denied chest pain, syncope, palpitations, shortness of breath, and fever. His home medications included amphetamine and dextroamphetamine (Adderall XL), $30 \mathrm{mg}$ daily; montelucast (Singulair), $10 \mathrm{mg}$ daily; loratadine (Claritin), $10 \mathrm{mg}$ daily; and doxycycline, $100 \mathrm{mg}$ daily for acne. Physical examination revealed an irregularly irregular heartbeat at 160 beats per minute with no murmurs. ECG showed chaotic atrial tachycardia/atrial fibrillation with rapid ventricular response (Figure 3). Blood ethanol level was $155 \mathrm{mg} / \mathrm{dl}$. Cardiac enzymes were unremarkable, and serum electrolytes, thyroidfunction tests, and a lipid profile were normal. A cardiac ECG revealed a structurally normal heart without thrombus. Computed tomography of his brain was normal. The patient was given a bolus of $2 \mathrm{~L}$ of normal saline, and his heart rate responded by decreasing from 160 beats per minute to 90 to 110 beats per minute. He remained hemodynamically stable and was placed on a cardiac monitor overnight with continued intravenous fluid support. Approximately 12 hours after presentation, he spontaneously reverted to a normal sinus rhythm (Figure 4). He remained asymptomatic with a normal sinus rhythm during subsequent cardiology follow-up the next week.

Of note, the Division of Pediatric Cardiology at the Stony Brook University Medical Center has cared for two other cases of atrial fibrillation in healthy adolescents after excessive caffeine consumption in the past five years. These cases were not included in this series, as the patients were unable to be located to provide their consent.

\section{Discussion}

Soft drinks containing caffeine are the major source of caffeine intake in children and adolescents, and their caffeine consumption has risen exponentially in the last 30 years [8]. The fastest-growing trend is toward highly caffeinated beverages known as "energy drinks," which differ from "sports drinks" such as Gatorade ${ }^{\mathrm{TM}}$. The general public is unlikely to be educated about the amount of caffeine in energy drinks and the possible ill effects that these drinks may cause in children and adolescents who consume them [13]. Energy drinks contain three to four times the caffeine as a typical soda and promise to boost performance and to enhance metabolism. Energy drinks like Full Throttle ${ }^{\mathrm{TM}}$, Red Bull ${ }^{\mathrm{TM}}$, SoBe No Fear ${ }^{\mathrm{TM}}$, and Monster ${ }^{\mathrm{TM}}$ typically contain a combination of caffeine, carbohydrates, B vitamins, amino acids, and other ingredients. One 8.2-ounce can of Red Bull ${ }^{\mathrm{TM}}$ contains 


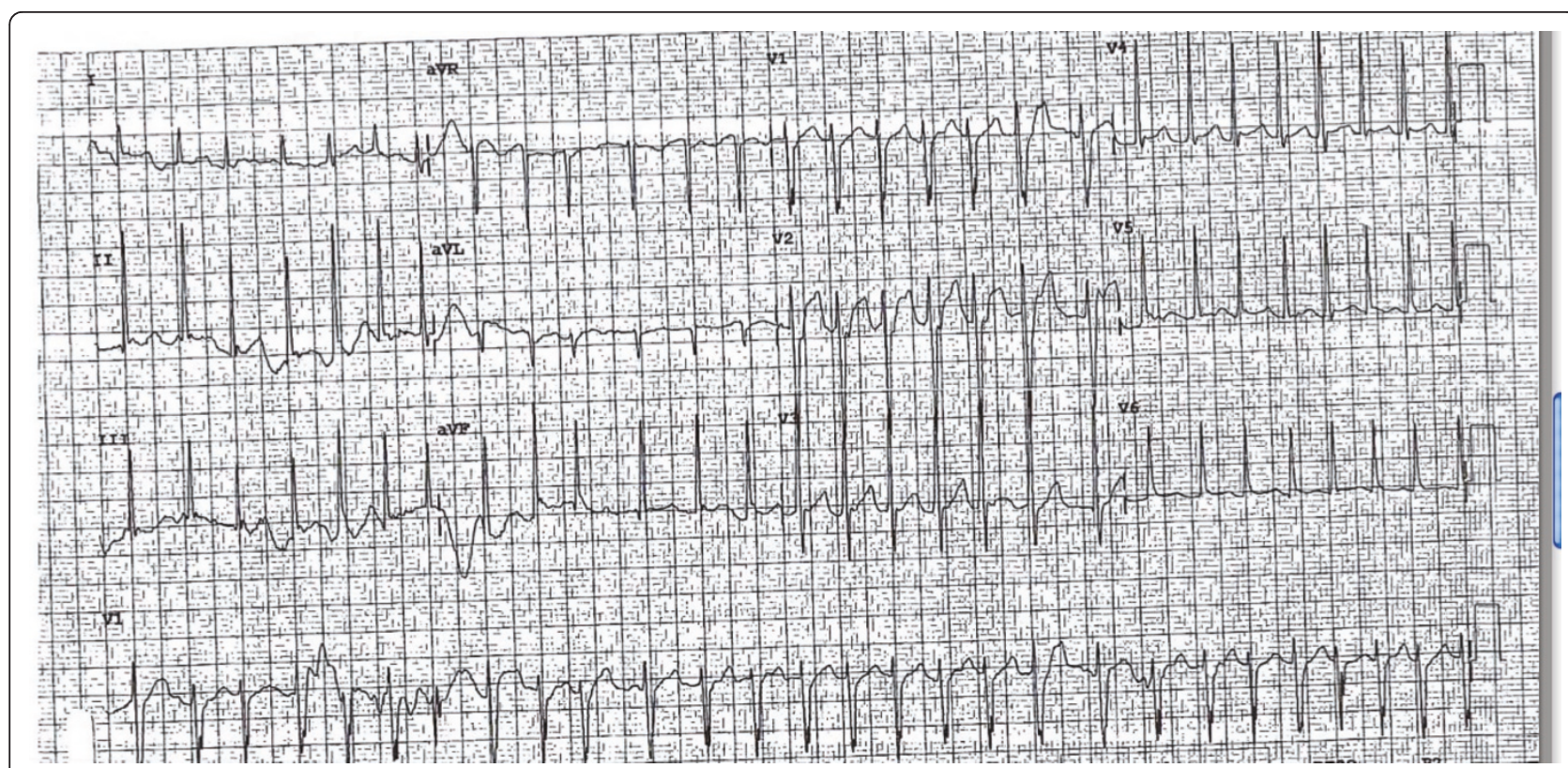

Figure 3 ECG showing chaotic atrial tachycardia/atrial fibrillation with rapid ventricular response at a rate of 166 beats per minute $\mathrm{QRS}, 97 \mathrm{~ms} ; \mathrm{QT} \mathrm{c}_{1} 492 \mathrm{~ms}$.

$80 \mathrm{mg}$ of caffeine $(0.03 \%)$, twice as much as a 12 -ounce soda; and one 16-ounce can of SoBe No Fear ${ }^{\mathrm{TM}}$ contains $141 \mathrm{mg}$ of caffeine, four times as much as a soda. Mountain Dew ${ }^{\mathrm{TM}}$, which is marketed along with other sodas, contains more caffeine than other typical sodas at $55 \mathrm{mg}$ per 12 ounces (Figure 5) [14,15]. Little regulation occurs with the production and marketing of energy drinks in the United States of America, with caffeine content between energy drinks ranging from $50 \mathrm{mg}$ to $505 \mathrm{mg}$ per bottle [16]. With the lack of regulation and strong marketing campaign toward young male athletes, energy drinks are becoming a serious threat to adolescents and are postulated to have caused grave consequences in an Australian athlete [17].

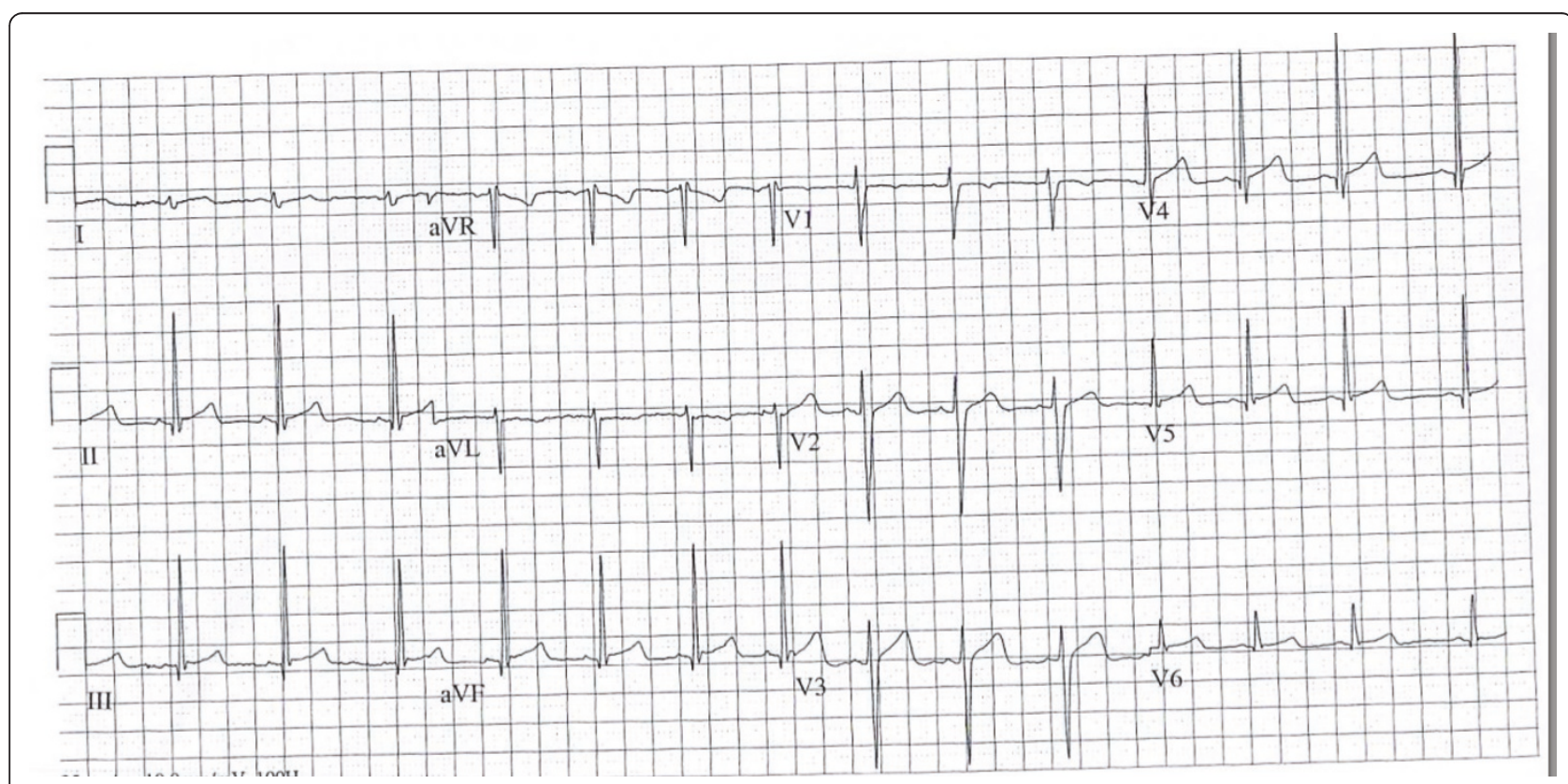

Figure 4 ECG showing restoration of normal sinus rhythm to 85 beats per minute after intravenous fluids. PR interval, 117 ms; QRS, 96 $\mathrm{ms} ; \mathrm{QT}_{\mathrm{c}}, 416 \mathrm{~ms}$. 


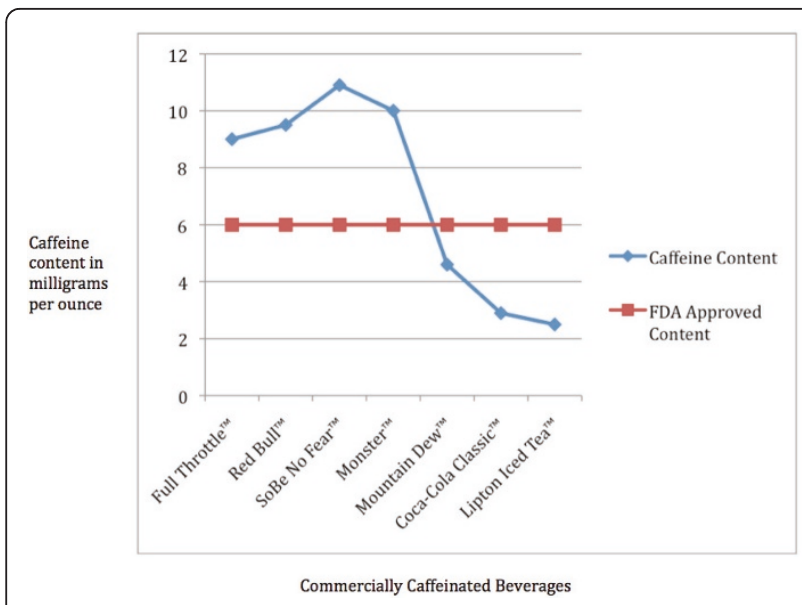

Figure 5 Caffeine content of common commercial beverages as compared with FDA-approved safe content of up to $0.02 \%$ (6 mg/ounce).

In our case series, both patients had essentially normal ECGs, ruling out endogenous cardiac causes for their arrhythmia, and both had admitted to consuming highly caffeinated drinks before atrial fibrillation developed. The patient in Case 1 admitted to consumption of a highly caffeinated beverage the day before his presentation, which was likely metabolized by the time he received medical care; one could argue that he may have had a prolonged caffeine half-life because of his chronic caffeine use. He did complain of the same palpitations after energy-drink consumption earlier that week; perhaps his high caffeine intake led to intermittent atrial fibrillation, which was exacerbated by his vigorous athletic activity on the day of presentation.

The patient in Case 2 had concurrent ingestion of alcohol with energy drinks and was also taking a baseline stimulant medication at the time of his presentation in atrial fibrillation. It is unclear how these other factors contributed to his arrhythmia; he certainly could have induced atrial fibrillation by alcohol intoxication, especially as his arrhythmia resolved with fluid resuscitation alone. As he did receive medical care within the expected half-life of caffeine, the timeline would fit for atrial fibrillation influenced by caffeine intoxication; the combination of alcohol and caffeine intoxication could have certainly led to his arrhythmia.

\section{Conclusion}

With the increasing popularity of energy drinks in the pediatric and adolescent population, physicians should be aware of the arrhythmogenic potential associated with their consumption. It is important for pediatricians to understand the lack of regulation in the caffeine content and other ingredients of these high-energy beverages and their complications, so that parents and children can be educated at well visits and sports physicals. We must inform the public on the potential health hazards related to excessive intake of caffeine-containing beverages by children and adolescents; the caffeine content of energy drinks should be better regulated and reported on food labels; and the purchase of energy drinks by the young consumer should be more closely monitored.

Given the possibility of cardiac arrhythmias and other untoward effects developing from caffeine use and abuse, further clinical trials reviewing the physiological effects and addictive potential for children and adolescents should be pursued, given the paucity of caffeine literature in this age group. Perhaps future studies could evaluate serum caffeine levels in pediatric patients who present with arrhythmias and concurrent caffeine consumption; this may be a useful measure to quantify into a risk model, should this correlation continue to be observed.

\section{Patient's Perspective}

"One night...I felt dizzy and lightheaded. I realized that my heart was beating abnormally fast. My chest felt alien to me because my heart was beating with no set rhythm and was shifting around inside my rib cage. I thought I had somehow knocked my heart loose from its rightful place and now it was swinging about inside my body, beating erratically. I was admitted to the intensive care unit...I was amazed at the professional intensity with which the doctors and nurses performed their duties. The cardiologist told us it could be a fluke occurrence maybe caused by sugar or caffeine intake. I was put on a drug through my IV. The next morning... (my heart rate) had dropped to normal...I was instantly in a more affable mood...Some may say this was the spark that ignited a fire inside me to pursue a career in the medical field."

\section{Consent}

Written informed consent has been obtained from the patient and parent of a patient who was a minor at the time for publication of this case report and accompanying images. Copies of the written consents are available for review by the Editor-in-Chief of this journal.

\section{Author details}

${ }^{1}$ The Medical College of Wisconsin, Pediatric Hospital Medicine, Suite C560, CCC, P.O. Box 1997, Milwaukee, WI, 53201-1997, USA. ²Beth Israel Medical Center, 1st Ave at 16th Street, New York, NY, 10003, USA. ${ }^{3}$ Stony Brook University Department of Pediatric Cardiology, HSC T-11, 040, Stony Brook, NY, 11794-8111, USA.

\section{Authors' contributions}

JRDR compiled, edited, and wrote the cases and also wrote the abstract, introduction, and discussion, and performed an updated review of the literature. AD initially summarized two cases, performed a literature review, 
and contributed to the introduction and discussion. PM was heavily involved in caring for the patients, selecting them for the case report and editing the manuscript; MBH and TAB were both directly involved in the care of the patients, helped select them for this case report series, and remained supportive of the manuscript and its editing. All authors read and approved the final manuscript.

\section{Competing interests}

The authors declare that they have no competing interests.

Received: 17 November 2009 Accepted: 19 January 2011

Published: 19 January 2011

\section{References}

1. Nanthakumar K, Lau YR, Plumb VJ: Electrophysiological findings in adolescents with atrial fibrillation who have structurally normal hearts. Circulation 2004, 110:117-123

2. Dubin A: Cardiac Arrhythmias: Nelson's Textbook of Pediatrics.Edited by: Kliegman et al. Philadelphia: Saunders Elsevier; , 18 2007:1942-1947.

3. Frost $L$, Vestergaard P: Caffeine and risk of atrial fibrillation or flutter: the Danish diet, cancer, and health study. Am J Clin Nutr 2005, 81:578-582.

4. Rashid A, Hines M, Scherlag BJ, Yamanashi WS, Lovallo W: The effects of caffeine on the inducibility of atrial fibrillation. J Electrocardiol 2006, 39:421-425.

5. Mehta A, Jain AC, Mehta MC, Billie M: Caffeine and cardiac arrhythmias: an experimental study in dogs with review of literature. Acta Cardiol 1997, 52:273-283.

6. Patanè S, Marte F, La Rosa FC, Rocca RL: Atrial fibrillation associated with chocolate intake abuse and chronic salbutamol inhalation abuse. Int J Cardiol 2009, E pub.

7. Peake STC, Mehta PA, Dubrey SW: Atrial fibrillation-related cardiomyopathy: a case report. J Med Case Rep 2007, 1:111.

8. Temple JL: Caffeine use in children: what we know, what we have left to learn, and why we should worry. Neurosci Biobehav Rev 2009, 33:793-806.

9. Magkos F, Kavouras S: Caffeine use in sports, pharmacokinetics in man, and cellular mechanisms of action. Crit Rev Food Sci Nutri 2005, 45:535-562.

10. Kaplan GB, Greenblatt DJ, Ehrenberg BL, Goddard JE, Cotreau MM, Harmatz JS, Shader RI: Dose-dependent pharmacokinetics and psychomotor effects of caffeine in humans. J Clin Pharm 1997, 37:693-703.

11. FDA Basics: "Why isn't the amount of caffeine a product contains required on a food label?". U.S. Food and Drug and Administration; [http://www.fda.gov]

12. Malinauskas BM, Aeby VG, Overton RF, Carpenter-Aeby T, Barber-Heidal K: A survey of energy drink consumption patterns among college students. J Nutr 2007, 6:35.

13. O'Dea J: Consumption of nutritional supplements among adolescents: usage and perceived benefits. Health Ed Res 2003, 18:98-107.

14. McCusker RR, Goldberger BA, Cone EJ: Caffeine content of energy drinks, carbonated sodas, and other beverages. J Analyt Toxicol 2006, 30:112-114.

15. The Caffeine Database. [http://www.energyfiend.com/the-caffeinedatabase].

16. Reissig CJ, Strain EC, Griffiths RR: Caffeinated energy drinks: a growing problem. Drug Alcohol Dep 2009, 99:1-10.

17. Berger AJ, Alford K: Cardiac arrest in a young man following excess consumption of caffeinated "energy drinks". Med J Aust 2009, 190:41-43.

doi:10.1186/1752-1947-5-18

Cite this article as: Di Rocco et al:: Atrial fibrillation in healthy adolescents after highly caffeinated beverage consumption: two case reports. Journal of Medical Case Reports 2011 5:18.

\section{Submit your next manuscript to BioMed Central and take full advantage of:}

- Convenient online submission

- Thorough peer review

- No space constraints or color figure charges

- Immediate publication on acceptance

- Inclusion in PubMed, CAS, Scopus and Google Scholar

- Research which is freely available for redistribution

Submit your manuscript at www.biomedcentral.com/submit
Biomed Central 